\title{
Tofacitinib Associated with Reduced Intubation Rates in the Management of Severe COVID-19 Pneumonia: A Preliminary Experience
}

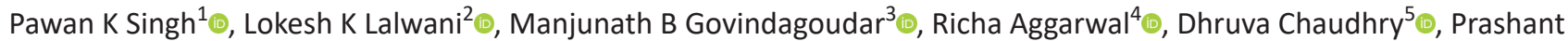 \\ Kumar $^{6}$, Preeti Gehlaut $^{7}$
}

\begin{abstract}
Background: The second wave of COVID-19 pandemic was not only associated with a rapid and severe surge in the number of cases but also limited availability of recommended medicines. Baricitinib has been known to reduce recovery time in COVID-19 pneumonia in association with remdesivir. Tofacitinib, with limited evidence, was used in severe COVID-19 pneumonia based on its similarity of action with baricitinib. Methods: Data of all patients admitted to the COVID-19 intensive care unit in the month of April were accessed and analyzed. Data of patients who were on other immunomodulators, invasive ventilation, or suffering from end-stage organ diseases were excluded from the analysis.

Results: Out of 73 patients, data of 50 were analyzed. Twenty-five received tofacitinib and the other 25 were managed with standard of care. Age, comorbidities, and gender distribution between the two groups were similar. On day 7 of admission, the change in $\mathrm{SpO}_{2} / \mathrm{FiO}_{2}$ ratio was $1.26 \pm 1$ and $0.72 \pm 1$ in the tofacitinib group and control group, respectively. Similarly, a higher number of subjects in the control group showed worsening in the World Health Organization (WHO) ordinal scale (36 vs $12 \%, p=0.01$ ). The clinical objective improvement was similar in the two groups. The intubation rates in the tofacitinib group were significantly lower than that in the control group ( $32 \%$ vs $8 \%, p=0.034)$. Conclusion: Tofacitinib, in this retrospective single-center experience, was found to be associated with reduced intubation rates and reduced worsening in the WHO ordinal scale. There was no difference in mortality in the two groups.

Keywords: COVID-19 pandemic, COVID-19, JAK/STAT, Severe COVID, Tofacitinib.

Indian Journal of Critical Care Medicine (2021): 10.5005/jp-journals-10071-23964
\end{abstract}

\section{HIGHLIGHTS}

Tofacitinib, a JAK/STAT inhibitor, has been used in COVID-19 pneumonia in anecdotal reports. In this report, we describe our experience with tofacitinib, in the background of nonavailability of baricitinib and tocilizumab. Our results indicate that tofacitinib is associated with clinically relevant outcomes, like reduced intubation rates and halting the worsening respiratory failure.

\section{INTRODUCTION}

COVID-19 pandemic, especially the second wave, has wreaked havoc across the country. The huge surge in the number of cases has been accompanied by an acute shortage of drugs, which have a proven role in severe COVID-19 disease. There are multiple factors that have been attributed to the limited drug availability, like decreased production, delayed supply, and hoarding. Among the recommended drugs for management of COVID-19, few important and mortality-reducing drugs are steroids, baricitinib, and to some extent remdesivir and tocilizumab. As the most important cause for mortality in COVID-19 disease is hypoxemic respiratory failure secondary to cytokine storm and lung involvement, most of the trials have attempted immunomodulatory therapies for reducing inflammation and halting the cytokine storm. Among these drugs, the most studied are tocilizumab ${ }^{1}$ and baricitinib. ${ }^{2}$ The Center for Disease Control and Prevention, the United States of America, has recommended the timely use of these drugs. But due to the limited availability and exponential spurt of cases, the treating physicians and intensivists were pushed to the wall and had to innovate while dealing with cytokine storm. Network theoretic analysis of
1-3,5 Department of Pulmonary and Critical Care Medicine, PGIMS, Rohtak, Haryana, India

${ }^{4}$ Department of Internal Medicine, PGIMS, Rohtak, Haryana, India

${ }^{6,7}$ Department of Anesthesiology and Critical Care, PGIMS, Rohtak, Haryana, India

Corresponding Author: Manjunath B, Govindagoudar, Department of Pulmonary and Critical Care Medicine, PGIMS, Rohtak, Haryana, India, Phone: +91 7015212965, e-mail: bgmanzu@gmail.com

How to cite this article: Singh PK, Lalwani LK, Govindagoudar MB, Aggarwal R, Chaudhry D, Kumar P, et al. Tofacitinib Associated with Reduced Intubation Rates in the Management of Severe COVID-19 Pneumonia: A Preliminary Experience. Indian J Crit Care Med 2021; 25(10):1108-1112.

Source of support: Nil

Conflict of interest: None

JAK/STAT pathway modulators has been hypothesized to have a significant role in halting the cytokine storm. Consistent with these theories, baricitinib, a JAK1 and JAK2 inhibitor, in association with remdesivir was found to be associated with reducing time to recovery in the landmark adaptive COVID-19 treatment trial $2 .^{2}$

One such drug, tofacitinib, is an early generation nonspecific JAK/STAT inhibitor with predominant action on JAK1 and JAK3. Tofacitinib works at the intracellular level by inhibiting the interleukin (IL)-15- and IL-6-induced phosphorylation of STAT proteins. Downstream production of IL-4, IL-6, IL-8, IL-17, IL-22, tumor necrosis factor, and interferon- $\gamma$ is inhibited by oral tofacitinib. ${ }^{3}$

(o) The Author(s). 2021 Open Access This article is distributed under the terms of the Creative Commons Attribution 4.0 International License (https://creativecommons. org/licenses/by-nc/4.0/), which permits unrestricted use, distribution, and non-commercial reproduction in any medium, provided you give appropriate credit to the original author(s) and the source, provide a link to the Creative Commons license, and indicate if changes were made. The Creative Commons Public Domain Dedication waiver (http://creativecommons.org/publicdomain/zero/1.0/) applies to the data made available in this article, unless otherwise stated. 
In view of this action, it has been approved for use in rheumatoid arthritis, ulcerative colitis, and psoriatic arthritis.

Cytokine profile modulated by tofacitinib is similar to that of baricitinib. Due to the limited availability of baricitinib and tocilizumab, it was decided, in our intensive care unit, that tofacitinib with analogous action will be used after verbal informed consent from the patient. The use of tofacitinib in COVID-19 is not novel to this study as Hayek et al. in their retrospective study have shown that the use of tofacitinib was associated with survival benefit. In their study, tofacitinib was used in 138 subjects of COVID-19 pneumonia. Compared with retrospective controls from the same center who were receiving only dexamethasone, patients who received tofacitinib were at $62 \%$ reduced odds of dying (odds ratio $=0.38,95 \%$ confidence interval $0.19-0.76$ ). Toxicity profile did not differ between the two groups. ${ }^{4}$ Authors in this study used $20 \mathrm{mg}$ of tofacitinib daily in two divided doses for 5 to 7 days. The regimen seems to be extrapolated from the recommendations for the management of moderate to severe ulcerative colitis. ${ }^{5}$ Based on the findings of Hayek et al., we started using tofacitinib in $10 \mathrm{mg}$ twice daily dosing in all severe cases of COVID-19 disease. In this report, we have presented our experience of 25 cases of severe COVID-19 who were managed with tofacitinib in comparison to their peers who received no immunomodulators apart from steroids.

\section{Methods and Materials}

\section{Study Setting and Design}

This was a retrospective case-control study done at a tertiary teaching hospital in north India. The study included participants who had received tofacitinib as an alternative to baricitinib or tocilizumab, both of which were not available for use. Fifty cases were reviewed of which 25 had received tofacitinib whereas the other 25 were managed as per management protocol issued by the ministry of health and family welfare (MoHFW-July 2020 version 5). Records for all patients who were admitted to our ICU in the month of April and May were accessed after due permission from the ethics committee.

\section{Participants}

All patients recruited in the analysis were confirmed cases of COVID-19 either with rapid antigen test or with RT-PCR for SARS-CoV-2 virus. All cases were on oxygen therapy either with a nonrebreathing face mask with reservoir, high-flow oxygen therapy, or noninvasive ventilation, at the time of admission. The etiology of respiratory failure was confirmed to be COVID-19 pneumonia through chest radiography or high-resolution computed tomography of the thorax. All participants belonged to severe category as per classification by MoHFW. Almost all subjects were within the first 15 days of illness. The presence of autoimmune comorbidities, malignancy, heart failure, liver failure, renal failure, and concomitant use of any other immunomodulatory therapy was considered to be prohibitory for the use of tofacitinib. Similarly, we excluded all the cases who were on invasive mechanical ventilation or the ones who were already on baricitinib or tocilizumab.

\section{Protocol}

Our hospital predominantly follows the clinical management protocol for COVID-19 released by MoHFW (version 5) on July 3, $2020 .^{6}$ In short, we uniformly use intravenous dexamethasone after initial use of methylprednisolone for 3 to 5 days, therapeutic anticoagulation, and remdesivir apart from the usual ICU care.
In steroid nonresponders, other immunomodulators are also considered. In the first week of April, the availability of the recommended immunomodulators (by the CDC), like baricitinib and tocilizumab, became restricted and other alternatives, like bevacizumab, itolizumab, and adalimumab, were being tried. In our hospital, based on the recent evidence and similarity of the mechanism of action with baricitinib, we started using tofacitinib. Tofacitinib was offered to the patients after explaining the possible mechanism of action in COVID-19 while keeping in mind the limited evidence and absence of clear-cut recommendations. Data of all the cases admitted to our ICU in the month of April, who fulfilled the eligibility criteria, were collected from hospital records. All patients were followed for 21 days since the admission or until discharge, whichever was earlier.

\section{Data Collected}

Apart from the demographic details, data regarding the current illness, past illness, and severity assessment were recorded for all patients. Instead of $\mathrm{PaO}_{2} / \mathrm{FiO}_{2}$ (partial pressure of arterial oxygen/ fraction of inspired oxygen), we used $\mathrm{SpO}_{2} / \mathrm{FiO}_{2}$ (oxygen saturation as measured by pulse oximetry/fraction of inspired oxygen), as it involves a noninvasive technique and can be assessed in real time. Also, previous studies have shown that $\mathrm{SpO}_{2} / \mathrm{FiO}_{2}$ correlates significantly with $\mathrm{PaO}_{2} / \mathrm{FiO}_{2}$ ratio. ${ }^{7}$

\section{Outcomes}

The World Health Organization (WHO), in their R \& D blueprint document, has advised for the use of ordinal scale as a primary end point for the assessment of clinical efficacy. ${ }^{8}$ In the same document, based on therapeutic role of investigational product, the secondary outcomes for efficacy as well as safety were also suggested, like viral clearance, levels of biomarkers inflammation, and treatment-emergent adverse effects. Other clinical markers of efficacy, like mortality, ICU stay, and hospital stay, were also advised. In concordance with the WHO document, we also used similar outcomes for our study. Tofacitinib is easily available as it is a commonly used Food and Drug Administration approved therapy for rheumatoid arthritis, ulcerative colitis, and psoriatic arthritis.

\section{Statistical Analysis}

Data were presented in a descriptive fashion as means (and standard deviation) or numbers (percentage with 95\% confidence intervals). The difference between continuous variables was analyzed using the Mann-Whitney $U$ (not normally distributed) and the Student's $t$-test (normally distributed). The difference between categorical variables was analyzed using Fischer's exact test. The $p$ values less than 0.05 were considered statistically significant. Statistical analysis was done using the IBM ${ }^{\circledast}$ SPSS $^{\circledast}$ software platform (version 26.0).

\section{Results}

From April 8 to May 10, a total of 73 patients were admitted to ICU with hypoxemic respiratory failure and COVID-19 pneumonia. Twenty-three subjects were excluded from the analysis for various reasons as mentioned in the consort chart (Fig. 1). Out of the remaining 50 subjects, 25 were treated with tofacitinib along with the standard of care (tofacitinib group), and the rest of the subjects were managed with the only standard of care. The demographic and clinical severity details of these subjects are presented in Table 1. Patients in the control group had a higher $\mathrm{SpO}_{2} / \mathrm{FiO}_{2}$ ratio but at the same time, a greater number of patients 
in the control group were in category 5 as per WHO ordinal scale [requiring noninvasive ventilation (NIV) or high-flow oxygen therapy(HFOT)], but both parameters were not statistically significant. Age, gender, and comorbidities status were not significantly different between the two groups. Due to significant hypoxia, we were not able to record the anthropometric measures of the patients and hence obesity was not evaluated in the two groups. Day of illness at the time of presentation was 10.5 and 12.8 days in the tofacitinib and control group, respectively. The majority of the computed tomographies of thorax were done much early in the course of illness, and the computed tomography severity index was similar in the two groups.

As far as concomitant medicines are concerned, all patients in both groups were managed with dexamethasone and anticoagulants apart from the usual ICU care (Table 2). In addition to this, remdesivir was used in 52 and $72 \%$ of the subjects in the tofacitinib group and control group, respectively. Plasma therapy was used in one subject in tofacitinib group, on compassionate grounds, on day 14 of admission, who did not show improvement in respiratory parameters. In the same subject, tofacitinib was withheld from day 10 of admission. Tocilizumab was tried in three subjects in the control group and one subject in the tofacitinib

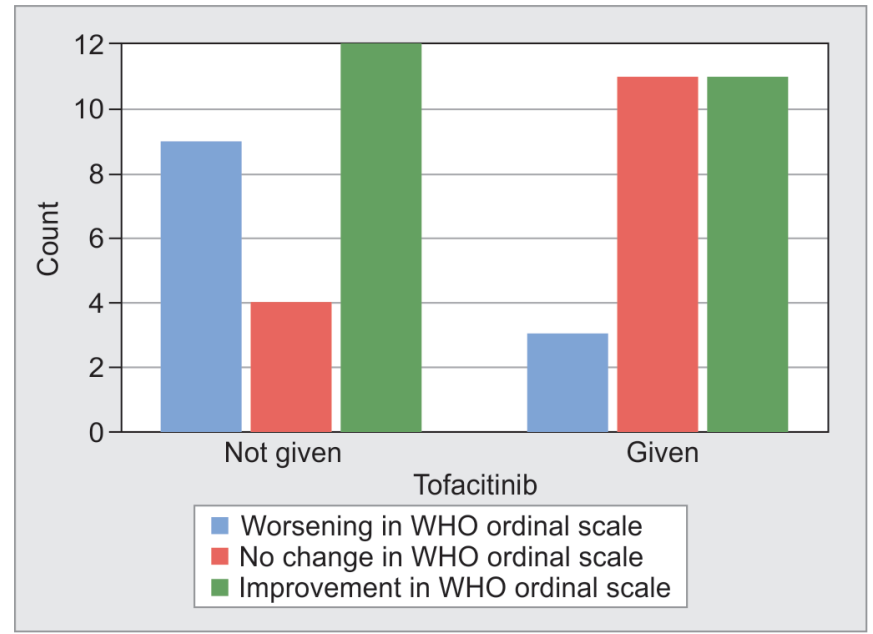

Fig. 1: Consort diagram showing flow of subjects group. One out of three subjects who received tocilizumab in the control group expired, and the subject in the tofacitinib group who had received tocilizumab also expired. In selected patients of COVID-19 severe disease, our hospital follows a policy of using injectable methylprednisolone therapy $(125 \mathrm{mg})$ in the early stages of illness (for 3-5 days) switching over to dexamethasone subsequently ( 56 vs $68 \%$ in tofacitinib group vs control group).

In efficacy data (Table 3 ), there was a fall in requirement of oxygen supplementation in the tofacitinib group as shown by the $\mathrm{FiO}_{2}$ requirement on day 7 (41.8 vs $49.2 \%, p=0.36$, tofacitinib vs control group, respectively) to maintain a similar oxygen saturation, albeit it was not statistically significant. Similarly, $\mathrm{SpO}_{2} / \mathrm{FiO}_{2}$ ratio was higher in the tofacitinib group on day 7 as compared to the control group but it was also not statistically significant. We also tried to evaluate the numeric change in $\mathrm{SpO}_{2} / \mathrm{FiO}_{2}$ from day 1 to day 7 and the change was higher in the tofacitinib group with a trend toward significance. Similarly, in the WHO ordinal scale, a higher number of patients in the control group belonged to category 6 (intubated and on mechanical ventilation) on day 7 . In the tofacitinib group, 12 patients were in category 5 requiring NIV or HFOT on day 1 , out of these 9 continued to be on NIV/HFOT, two got intubated and 1 improved by day 7 . In the control group, most of the subjects belonged to category 4 or 6 by day 7. Significantly higher number of patients in control group got intubated by day 7 (32 vs $8 \%, p=0.011)$. We also evaluated the change in the WHO ordinal scale from baseline. Nearly equal number of cases showed improvement in both groups but worsening as per WHO ordinal scale was statistically more common in the control group ( 36 vs $12 \%, p=0.043$ ). A higher number of cases

Table 2: Details of the use of concomitant medications in both groups

\begin{tabular}{lcc}
\hline & Tofacitinib group & Control group \\
\hline Remdesivir & $52 \%$ & $72 \%$ \\
Plasma therapy & $4 \%$ & 0 \\
Tocilizumab & $4 \%$ & $12 \%$ \\
$\begin{array}{l}\text { Dexamethasone } \\
\text { Methylprednisolone pulse }\end{array}$ & $100 \%$ & $100 \%$ \\
therapy & $56 \%$ & $68 \%$ \\
\hline
\end{tabular}

Table 1: Demographic and clinical profile of the patients

\begin{tabular}{|c|c|c|}
\hline & Tofacitinib group & Control group \\
\hline Gender (Male \%/Female \%) & $64 \% / 36 \%$ & $64 \% / 36 \%$ \\
\hline Age (years) mean \pm SD & $45.52 \pm 15.67$ & $46.72 \pm 11.2$ \\
\hline Comorbidities (DM/HTN) \% & $56 \% / 40 \%$ & $64 \% / 44 \%$ \\
\hline Day of illness at the time of presentation mean \pm SD & $10.5 \pm 2.7$ & $12.8 \pm 3.6$ \\
\hline $\mathrm{SpO}_{2}$ at presentation (\%) mean $\pm \mathrm{SD}$ & $91.56 \pm 3.48$ & $92.44 \pm 3.61$ \\
\hline $\mathrm{FiO}_{2}$ at presentation (\%) mean $\pm \mathrm{SD}$ & $64.68 \pm 25.46$ & $56.64 \pm 23.82$ \\
\hline $\mathrm{SpO}_{2} / \mathrm{FiO}_{2}$ ratio & $1.75 \pm 0.97$ & $1.93 \pm 0.82$ \\
\hline CTSI [number of subjects done in/(mean score \pm SD)] & $16(15.2 \pm 6.0)$ & $18(14.0 \pm 5.2)$ \\
\hline WHO ordinal scale at presentation & $2(8 \%)$ & $1(4 \%)$ \\
\hline 3 (hospitalized without oxygen therapy) & $11(44 \%)$ & $8(32 \%)$ \\
\hline $\begin{array}{l}4 \text { (oxygen with mask or nasal prongs) } \\
5 \text { (NIV or HFNC) }\end{array}$ & $12(48 \%)$ & $16(64 \%)$ \\
\hline
\end{tabular}

$\mathrm{SD}$, standard deviation; $\mathrm{DM}$, diabetes mellitus; $\mathrm{HTN}$, hypertension; $\mathrm{SpO}_{2}$, oxygen saturation; $\mathrm{FiO}_{2}$, fraction of inspired oxygen; CTSI, computed tomography severity index; WHO, World Health Organization; NIV, noninvasive ventilation; HFNC, high-flow nasal cannula 
Table 3: Efficacy parameters of both groups

\begin{tabular}{|c|c|c|c|}
\hline & Tofacitinib group & Control group & pvalue \\
\hline $\mathrm{SpO}_{2}$ on day 7 & $93.6 \pm 2.2$ & $93.4 \pm 4.8$ & 0.85 \\
\hline $\mathrm{FiO}_{2}$ on day 7 & $41.8 \pm 26.8$ & $49.2 \pm 30.4$ & 0.36 \\
\hline $\mathrm{SpO}_{2} / \mathrm{FiO}_{2}$ on day 7 & $3.0 \pm 1.38$ & $2.6 \pm 1.4$ & 0.37 \\
\hline Change in $\mathrm{SpO}_{2} / \mathrm{FiO}_{2}$ ratio compared to day 1 & $1.26 \pm 1.0$ & $0.72 \pm 1.0$ & 0.073 \\
\hline $\begin{array}{l}\text { WHO ordinal scale on day } 7 \\
\qquad 2 \text { (discharged with activity limitation) }\end{array}$ & $20 \%$ & $8 \%$ & 0.011 \\
\hline 3 (hospitalized without oxygen therapy) & $20 \%$ & $16 \%$ & \\
\hline 4 (oxygen therapy with mask of nasal prongs) & $16 \%$ & $40 \%$ & \\
\hline 5 (NIV or HFNC) & $36 \%$ & $4 \%$ & \\
\hline 6 (intubated and mechanical ventilation) & $8 \%$ & $32 \%$ & \\
\hline $\begin{array}{l}\text { Change in WHO ordinal scale } \\
\text { Worsening }\end{array}$ & $12 \%$ & $36 \%$ & 0.043 \\
\hline Static & $44 \%$ & $16 \%$ & \\
\hline Improvement & $44 \%$ & $48 \%$ & \\
\hline Intubation on day 7 & $8 \%$ & $32 \%$ & 0.034 \\
\hline Death by day 21 & $12 \%$ & $20 \%$ & 0.44 \\
\hline
\end{tabular}

Mean value of oxygen saturation; Fraction of oxygen of both groups. $\mathrm{SpO}_{2}$, oxygen saturation; $\mathrm{FiO}_{2}$, fraction of inspired oxygen; WHO, World Health Organization; NIV, noninvasive ventilation; HFNC, high-flow nasal cannula

Flowchart 1: Change in WHO ordinal scale from day 1 to day 7

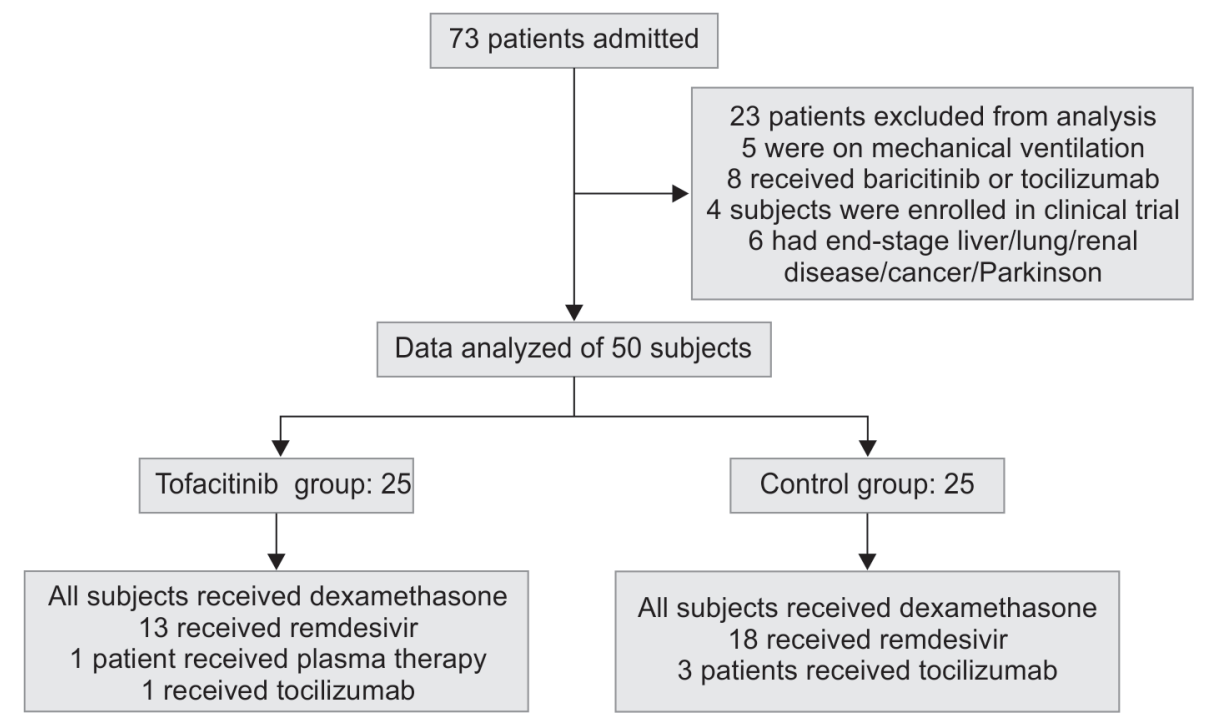

in the tofacitinib group continued to be in the same category on day 7 (44 vs 16\%) (Flowchart 1).

The clinically relevant outcomes of intubation and death were also evaluated. The tofacitinib group had a significantly lower number of intubations by day 7 as compared with the control group ( $32 \mathrm{vs} 8 \%, p=0.034$ ) but this did not reflect in the number of deaths by day 21 where the difference was not statistically significant (20 vs $12 \%, p=0.44)$.

There were no instances of new bacterial or invasive fungal infections or treatment-emergent adverse effects in either of the two groups.

\section{Discussion}

In this retrospective, single-center experience on the use of tofacitinib in severe COVID-19 pneumonia, we found that the

tofacitinib group had significantly lower intubation rates and a lower risk of worsening as per WHO ordinal scale by day 7 . There were no significant differences as per mortality and categorical improvement on the WHO scale between either of the two groups.

Despite over a year into the pandemic, COVID-19 remains a rapidly evolving disease. In the second wave of the pandemic, India witnessed new kinds of limitations in treatment and care, which were more of logistical as compared to the dearth of evidence. The meteoric rise in the number of cases was followed by a catastrophic fall in the availability of drugs, like remdesivir, tocilizumab, baricitinib, and oxygen. ${ }^{9}$ There are only four medicines in COVID-19, which have a robust backing up of evidence. Among them, the most important is dexamethasone, which has shown a significant reduction in 28-day mortality among hospitalized patients. ${ }^{10}$ At the same time, a significant number of patients either do not respond to 
dexamethasone only or have severe adverse events due to the same, like hyperglycemia, oral candidiasis, or bacterial infections. This led to an evaluation of various other immunomodulators, like tocilizumab and baricitinib. Tocilizumab is extensively studied in literature and has been found to have clinical benefits early in the course of illness in carefully selected few subjects. ${ }^{1,11}$ Similarly, baricitinib when given with remdesivir in hospitalized cases of COVID-19 was associated with a significant reduction in recovery time. Patients receiving baricitinib had a median recovery time of 7 days as compared to 8 days in the control group. ${ }^{12}$

Considering immunomodulation as an important component of armamentarium against COVID-19 pneumonia, various other drugs have been evaluated in COVID-19, like itolizumab, bevacizumab, and adalimumab with limited evidence. ${ }^{13}$ Given the fact that baricitinib is the only drug with the strongest positive evidence, we were inclined to use similar congeners of baricitinib. Among them, there is limited but positive evidence for tofacitinib. ${ }^{14}$ The influence of tofacitinib on the cytokine profile of subjects is not only similar to baricitinib but also broader. Being pushed to the wall with limited availability of medication, we started using tofacitinib and observed significant clinical benefits of the same. With the rise in cases of mucormycosis in COVID-19 patients, who have been managed with dexamethasone, there is an urgent need for metabolically neutral and broad-acting immunomodulators.

Evidence generation for or against any therapy is a long and rigorous task, time for which was not allowed by the pandemic. This is reflected in the limited number of robust trials being available for COVID-19. ${ }^{15}$ Tofacitinib is proven to be a safe medicine with limited short-term adverse effects. ${ }^{16}$ Given the lack of availability of proven medicine, our findings present evidence in favor of the use of tofacitinib in severe cases of COVID-19 pneumonia, presenting with hypoxemic respiratory failure.

There are several limitations to our study, like being a singlecenter, retrospective, and open-label experience due to which the index study is bound to have an inherent bias. In addition to this, we did not focus on analyzing the effect of immunomodulation on inflammatory markers, like C-reactive protein and ferritin. Also, we excluded most of the cases who had received other immunomodulators, like baricitinib and tocilizumab. The number is not large enough to conclusively derive evidence apart from being hypothesis-generating. Had the number of cases been large enough with a longer follow-up, we could have found out the effect of tofacitinib on mortality as well. Despite these limitations, this is the first Indian study describing the use of tofacitinib in COVID-19 pneumonia. The answers to these questions and limitations will be provided by the phase 2/3 TOSCO (TOfacitinib for Severe COVID-19 disease) trial, which is currently ongoing.

\section{Conclusion}

In conclusion, we have presented here the preliminary experience of tofacitinib in COVID-19. We have found that tofacitinib is associated with a significant reduction in intubation rates and prevention of clinical worsening as per the WHO ordinal scale. This limited data call for a randomized controlled trial for the use of tofacitinib in severe COVID-19. It is also prudent to evaluate the efficacy of tofacitinib against the commonly used medications, like baricitinib, tocilizumab, and dexamethasone.

\section{OrCID}

Pawan K Singh (1) https://orcid.org/0000-0002-3212-9875

Lokesh K Lalwani @ https://orcid.org/0000-0002-7652-309X

Manjunath B Govindagoudar (1) https://orcid.org/0000-0003-2704 $-0873$

Richa Aggarwal @ https://orcid.org/0000-0002-0237-0094

Dhruva Chaudhry (i) https://orcid.org/0000-0001-5138-2908

Prashant Kumar (1) https://orcid.org/0000-0002-6460-8563

Preeti Gehlaut (1) https://orcid.org/0000-0002-1185-9270

\section{References}

1. Soin AS, Kumar K, Choudhary NS, Sharma P, Mehta Y, Kataria S, et al. Tocilizumab plus standard care versus standard care in patients in India with moderate to severe COVID-19-associated cytokine release syndrome (COVINTOC): an open-label, multicentre, randomised, controlled, phase 3 trial. Lancet Respir Med 2021;9(5):511-521. DOI: 10.1016/S2213-2600(21)00081-3.

2. Kalil AC, Patterson TF, Mehta AK, Tomashek KM, Wolfe CR, Ghazaryan V, et al. Baricitinib plus remdesivir for hospitalized adults with covid-19. New Engl J Med 2020;384(9):795-807. DOI: 10.1056/NEJMoa2031994.

3. Hodge JA, Kawabata TT, Krishnaswami S, Clark JD, Telliez JB, Dowty ME, et al. The mechanism of action of tofacitinib - an oral Janus kinase inhibitor for the treatment of rheumatoid arthritis. Clin Exp Rheumatol 2016;34(2):318-328. www.clinexprheumatol.org/abstract.asp?a=9615

4. Hayek ME, Mansour M, Ndetan H, Burkes Q, Corkren R, Dulli A, et al. Anti-inflammatory treatment of COVID-19 pneumonia with tofacitinib alone or in combination with dexamethasone is safe and possibly superior to dexamethasone as a single agent in a predominantly African American cohort. Mayo Clin Proc Innov Qual Outcomes 2021;5(3):605-613. DOI: 10.1016/j.mayocpiqo.2021.03.007.

5. Panés J, Gisbert JP. Efficacy of tofacitinib treatment in ulcerative colitis. Gastroenterol Hepatol 2019;42(6):403-412. DOI: 10.1016/j. gastrohep.2019.03.002.

6. Welfare GolMoHaF. Clinical management protocol: covid-19. In: Services DGoH, editor. New Delhi: MoHFW; 2020. p. 23.

7. Rice TW, Wheeler AP, Bernard GR, Hayden DL, Schoenfeld DA, Ware LB. Comparison of the $\mathrm{SpO}_{2} / \mathrm{FiO}_{2}$ ratio and the $\mathrm{PaO}_{2} / \mathrm{FiO}_{2}$ ratio in patients with acute lung injury or ARDS. Chest 2007;132(2):410-417. DOI: 10.1378/chest.07-0617.

8. Herson J. Clinical trial preparations for the next pandemic. Contemp Clin Trials 2021;102:106292. DOI: 10.1016/j.cct.2021.106292.

9. Bhattacharjee S. Drug shortage hits patients. The Hindu 2021;Sect. Cities.

10. Horby P, Lim WS, Emberson JR, Mafham M, Bell JL, Linsell L, et al Dexamethasone in hospitalized patients with covid-19. N Engl J Med 2021;384(8):693-704. DOI: 10.1056/NEJMoa2021436.

11. Chaudhry D, Singh PK. Tocilizumab and COVID-19. Indian J Crit Care Med 2020;24(9):741-743. DOI: 10.5005/jp-journals-10071-23608.

12. Kalil AC, Patterson TF, Mehta AK, Tomashek KM, Wolfe CR, Ghazaryan V et al. Baricitinib plus remdesivir for hospitalized adults with covid-19. New Engl J Med 2021;384(9):795-807. DOI: 10.1056/NEJMoa2031994.

13. Rizk JG, Kalantar-Zadeh K, Mehra MR, Lavie CJ, Rizk Y, Forthal DN Pharmaco-immunomodulatory therapy in COVID-19. Drugs 2020;80(13):1267-1292. DOI: 10.1007/s40265-020-01367-z.

14. Banerjee A, Goswami RP, Chatterjee M. Network theoretic analysis of JAK/STAT pathway and extrapolation to drugs and viruses including COVID-19. Sci Rep 2021;11(1):2512. DOI: 10.1038/s41598-021-82139-x.

15. Nicola M, O'Neill N, Sohrabi C, Khan M, Agha M, Agha R. Evidence based management guideline for the COVID-19 pandemic - review article. Int J Surg 2020;77:206-216. DOI: 10.1016/j.ijsu.2020.04.001.

16. Tofacitinib. Drugs and Lactation Database (LactMed). Bethesda (MD): National Library of Medicine (US); 2006. 